\title{
Development of a Chirp Stimulus PC-Based Auditory Brainstem Response Audiometer
}

\author{
Ali AL-Afsaa ${ }^{1,2}$ \& Soegijardjo Soegijoko ${ }^{2}$ \\ ${ }^{1}$ Homs Health Care Department; Homs-Syria. E-mail:syrianjaya@hotmail.com \\ ${ }^{2}$ Biomedical Engineering Program, Department of Electrical Engineering, Institut \\ Teknologi Bandung. Jalan Ganesha 10, Bandung 40132, Indonesia. Phone/Fax (62)-22- \\ 2534117; E-mail: biomed@ee.it.ac.id.
}

\begin{abstract}
Hearing losses during infancy and childhood have many negative future effects and impacts on the child life and productivity. The earlier detection of hearing losses, the earlier medical intervention and then the greater benefit of remediation will be. During this research a PC-based audiometer is designed and, currently, the audiometer prototype is in its final development steps. It is based on the auditory brainstem response (ABR) method. Chirp stimuli instead of traditional click stimuli will be used to invoke the ABR signal. The stimulus is designed to synchronize the hair cells movement when it spreads out over the cochlea. In addition to the available hardware utilization (PC and PCI board), the efforts confined to design and implement a hardware prototype and to develop a software package that enables the system to behave as ABR audiometer. By using such a method and chirp stimulus, it is expected to be able to detect hearing impairment (sensorineural) in the first few days of the life and conduct hearing test at low frequency of stimulus. Currently, the intended chirp stimulus has been successfully generated and the implemented module is able to amplify a signal (on the order of ABR signal) to a recordable level. Moreover, a NI-DAQ data acquisition board has been chosen to implement the PC-prototype interface.
\end{abstract}

Keywords: ABR audiometer, audiometry, chirp stimulus, Hearing impairments, low frequency hearing test, PC-based audiometer, sensorineural disorder, Under-fives Hearing test.

\section{$1 \quad$ Introduction}

It is difficult for human of normal hearing-sense to feel how bitter the life is without hearing sense, but they can imagine how their life will be if they were born deaf. According to the National Institutes of Health Consensus Development Conference Statement some children in USA, approximately 1 of every 1000, are born deaf. Many more are born with less severe degrees of hearing impairment, while others develop hearing impairment during childhood (NIH Consens 1993). Reduced hearing acuity during infancy and early childhood interferes with the development of speech and verbal language skills. Significantly reduced auditory input adversely affects the developing auditory 
nervous system and can have harmful effects on social, emotional, cognitive, and academic development. Moreover, delayed identification and management of severe to profound hearing impairment may impede the child's ability to adapt his life in a hearing world or in the deaf community.

Audiometer is an equipment used to test the hearing system functionality and hearing acuity. ABR is one of several available methods to detect hearing dysfunctions. The ABR-based audiometers differ as the stimulus type they use differs. One or more of stimulus types (click, pure tone, pips, train pulse,.. etc) is used in available ABR audiometer. Different stimulus characteristics give different ABR audiometer hence each laboratory has its own specifications and characteristics and the manufacturers approve their product in comparison with behavioral method that is the traditional and most precise method for hearing impairment detection (Michael et, 1987; ANSI working group, 1998). Up to date, audiologists using available ABR audiometers are: first unable to detect hearing losses, disorders, and difficulties at frequency less than $1 \mathrm{kHz}$. It is because they use audiometers based on click stimulus to invoke recordable ABR response. Second, they are unable to distinguish severe-profound hearing losses in the range of 85-95 dB HL (hearing level) from those in the more profound ranges of 100-120 dB HL, especially at $500 \mathrm{~Hz}$. Third, they can conduct functional tests of the hearing system (normal or abnormal function) but not anatomy test (no indications to site of lesion). Chirp stimulus will be used throughout this research because it has already found that it can invoke ABR signal as good as the ABR signal of other kind of stimuli (Wegner, Dau and Kollmeier). Chirp stimulus is designed to synchronize the hair cells movement over the cochlea. It is in a way that forces the hearing cell to fire their electrical potential simultaneously. The acoustic stimuli stimulate the under test hearing system invoking ABR biopotenial. The standard EEG surface electrodes located on the scalp will pick up the acoustic evoked response. Then ABR will be conditioned and prepared analogically to fit the PC interface. The ABR data extraction and the advanced data process will be conducted by means of signal processing techniques.

Occurrence of hearing impairment at infancy and childhood has impacts on speech and language acquisition, academic achievement, and social/emotional development. The first three years of life is the most important for speech and language acquisition (NIH Consens 1993). Consequently, if a child is hard of hearing or deaf at birth or experiences hearing loss in infancy or early childhood, it is possible that child will not receive adequate auditory, linguistic, and social stimulation requisite to speech and language learning, social and emotional development, and that family functioning will suffer. There is a general agreement that hearing impairment should be recognized as early in life 
as possible. It is to have the full remediation advantage of the developing sensory system and to prevent or minimize the adverse effects.

The previous hearing test experiment has been conducted by using MatLab software and the Creative SB-Live card as a data acquisition board. The experiment failed to extract and record ABR signal because of the control shortage over the invoked ABR of repeated stimulus. An NI-DAQ data acquisition board from National Instruments is currently the prototype-computer interface. To use NI-DAQ board properly, prototype modifications have been conducted. Hence this article discusses the result of the recent progress of the conducted experiments.

At the end of research, it is expected to be able to record ABR signal over the whole frequency range ( 250 to $8000 \mathrm{~Hz}$ ). The recorded $\mathrm{ABR}$ is expected to include information related to the whole activated sensory hair cell in the cochlea.

\section{$2 \quad$ ABR Audiometers and Audiometry}

Audiometry is the process in which the audiologist conducts sensorineural hearing test by using an audiometer. Figure 1 shows a very simplified block diagram of ABR audiometer that works as follows. A stimulus generator generates audible stimuli of specific characteristics and repetition rate. An audio amplifier amplifies the generated stimuli to the level that produces user-selected loudness measured in decibel.

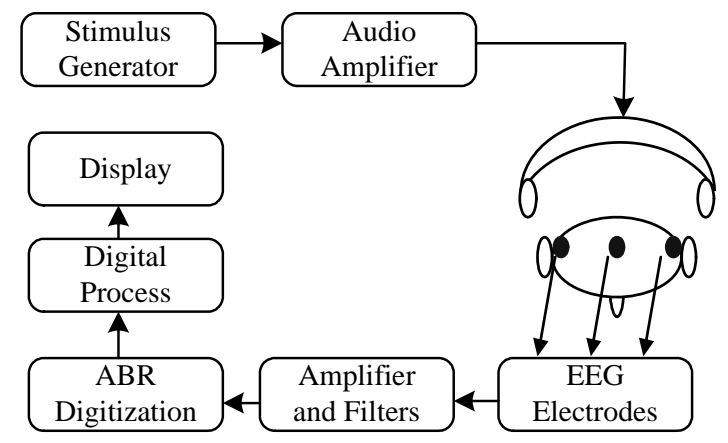

Figure 1 A simplified general block diagram of ABR audiometer.

A headset fixed to the patient head delivers the audible stimuli to the ear under test. Each stimulus evokes its own ABR potential that appears on the scalp. Standard EEG surface electrodes located on the scalp pick up the invoked ABR signal. ABR signal on the scalp of $0.1-10 \mu \mathrm{v}_{\mathrm{pp}}$ amplitude and has frequency components in the range of $100 \mathrm{~Hz}$ to $3 \mathrm{kHz}$ (Webster, G. Medical, 1998; Aston, 
1990). Picked up potentials are amplified, filtered, and conditioned analogically to attenuate environmental noise and the undesired biopotential components and enhance the required ABR signal. The signal is then digitized and processed digitally to extract ABR potential to be displayed and recorded.

\section{$3 \quad$ Ear Anatomy and Hearing Mechanism}

The ear can be divided, anatomically, into three parts they are: the outer, middle and inner ear. Very simply, the outer ear function is sound collection and localization. Its auditory canal ends at the tympanic membrane that changes the air pressure (sound) into mechanical vibration. The middle ear consists of three bones (malleus, incus and stapes) as shown in figure 2. It transmits the mechanical vibration of the tympanic membrane into the inner ear converting it into pressure wave in the fluid. The inner ear (cochlea) transduces sound vibrations in the fluid into electrical potential to be transmitted into the brain through the auditory nerve.

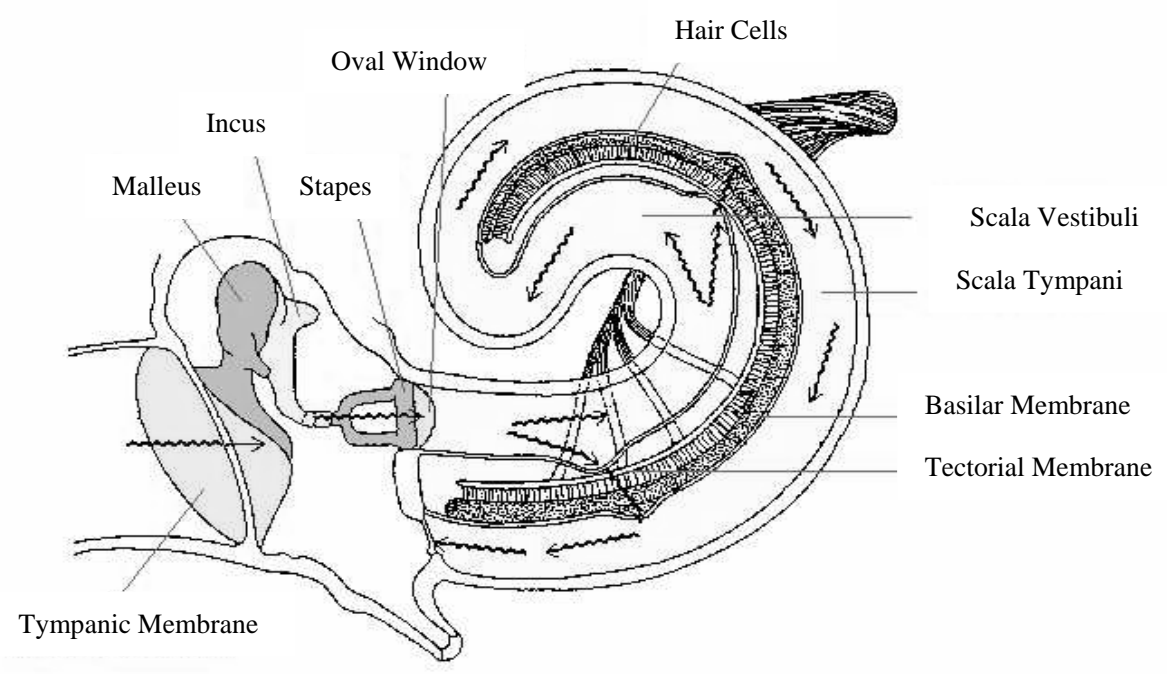

Figure 2 A simple representation of the cochlear and sound wave path (from Department of Anatomy, Tutorial, 2000).

The cochlea has a snail shape and complex structure. Two membranes divide the snail into three separate chambers: scala media, scala vestibula, and scala tympani (latest two chambers connected together by small window). The chambers are full of two different physiological liquids. The hair cells that are located on the basilar membrane in the scala media convert the sound vibration in the liquid into electrical potential. Depending on the hair cell frequency- 
sensitivity, the cochlea can be divided into different regions; each is sensitive to a different frequency (E.Glassock, Jackson, and Forrest Josey, 1987).

\section{$4 \quad$ Research Methodology}

Auditory Brainstem Response (ABR) signal has a few micro volts amplitude, i.e.0.1 to $10 \mu \mathrm{Vp}$-p. It will be picked up on the scalp where there exist other biopotential activities. They form the background noise. They have frequency components that fall in the same range as ABR components, but have minimum amplitude of the same order and greater. The environmental noise (especially $50 \mathrm{~Hz}$ of power lines) is the other source of noise. It has greater amplitude and may strongly interfere with acquired ABR signal.

In contrast to noise signals, ABR potential is time-locked because it appears as a result of stimulating the hearing system by an acoustic stimulus. Hence the averaging method becomes our reasonable choice to extract ABR signal from its background noise and to reduce the effect of other environmental noise signals that interfere randomly. ABR will be extracted by averaging 1000 stimulated ABRs at repetition rate of 2 stimuli per second. An analog high pass filter will effectively attenuate the $50 \mathrm{~Hz}$ noise and additional digital high pass filter will be to decrease the $50 \mathrm{~Hz}$ and its second and third harmonics.

Chirp stimulus characteristics will be designed to synchronize the hair cells movement leading to concurrent biopotentals that come from the all frequencysensitive regions of cochlea. Simultaneous firing of biopotentials is based on the different travel-time of different frequencies through different liquids. This is going to increase the amplitude and the information contents of stimulated ABR and can be useful to get regional cochlear information.

\section{$5 \quad$ System Design and Implementation}

Figure 3 shows a block diagram of the implemented and under development system. It consists of Personal Computer, PCI Data Acquisition Board, and Amp\&Control Circuit, Analog Process, and Power Supply (is not shown in the figure) modules. The PC uses a PCI data acquisition board (PCI 6024-E NiDAQ) to communicate with developed hardware.

A part of software package has been developed to generate a specific characteristics chirp stimulus. The stimulus is designed to have intensity (in $\mathrm{dB}$ ) that is entirely under software control. It is by setting up the analog output objects (channels) properties and assigning the variable amplitude value. The software is also used to design the user interface and to generate the required control commands. 


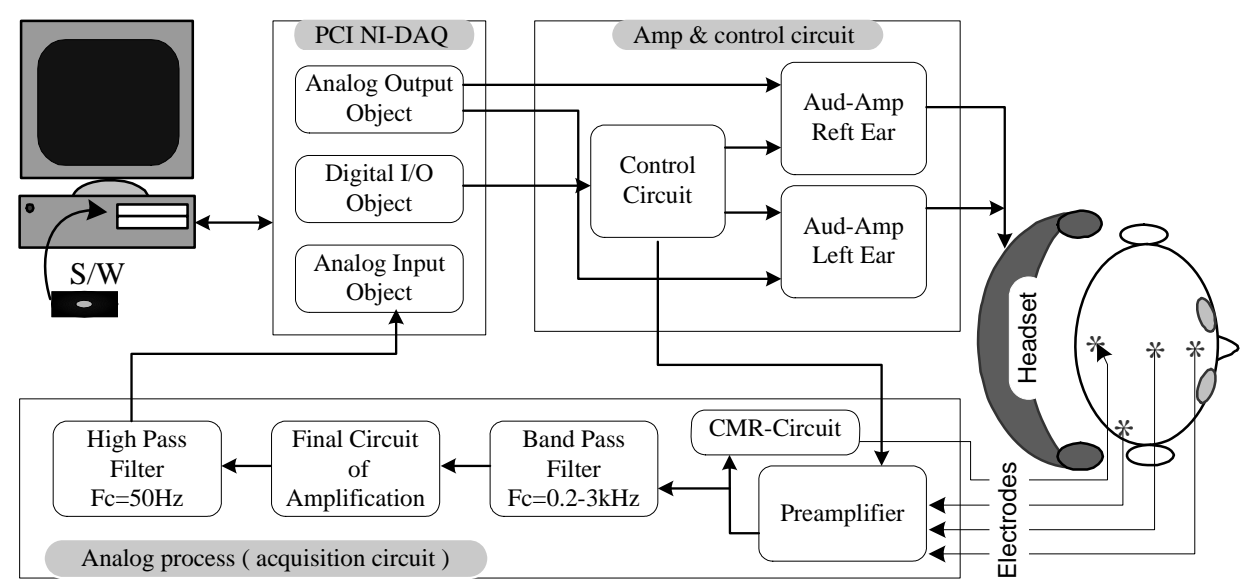

Figure 3 Block diagram of realized PC-based ABR Audiometer. The PCI NIDAQ board is presented outside the PC for clarity purpose.

Ni-DAQ has analog input, analog output, and digital input/output device objects. The board functionality and its input and output device objects setup are completely under software control. Ni-DAQ has been setup to provide two analog output channels. Each ear receives its stimulus from its dedicated channel as shown in figure 3. Each channel output has its own audio amplifier circuit that has been designed and implemented to have fixed gain of 2.4. The same output channels will be also used as synchronization signal output. It is software-generated signal and is necessary for conducting right timing between stimulus generation and response acquisition. Output channels, simultaneously and mutually, output the stimulus and synchronization signals. The control circuit has been implemented on the same board as audio amplifiers to translate the software-generated control commands. They are commands that determine the ear to be stimulated and then its electrode set that will pick up the invoked ABR response. The under test ear receives the amplified stimuli through the TDH39P headset. Two of the EEG surface electrodes located on the scalp are necessary to pick up the invoked ABR signal. They are switched to become the preamplifier circuit inputs. The circuit, primarily, amplifies picked up signal and passes it to a band-pass filter of $200-3000 \mathrm{~Hz}$ cutoff frequencies. The analog filters attenuate the biopotential and environment noise components that are outside the frequency range of $\mathrm{ABR}$ and at the same time enhances the ABR signal. During the final amplification and the $50 \mathrm{~Hz}$ high-pass filter stages, the ABR is amplified to be of the analog input level of Ni-DAQ and the $50 \mathrm{~Hz}$ is attenuated as much as possible. ABR signal is stored temporarily in the $\mathrm{Ni}$ DAQ registers before it is extracted to the PC memory where it is saved to a file for the later advanced process. 
In addition to control command generation, chirp stimulus generation, and user interface windows, the MatLab software will be used to conduct the required signal processing techniques to extract ABR signal information and to display and print the results

\section{Experimental Results}

The developed hardware prototype consists of three printed circuit boards (PCB). They are, as shown in figure 3, the acquisition circuit PCB, Aud-Amp \& control circuit PCB, and power supply which not included in figure 3 . The functionality of each circuit on those boards has been verified separately. Additional circuits, such as Trigger Circuit and Function Generator circuit (shown in figure 5), have been added for experimental purpose.

The control circuit has been designed an implemented to, automatically, apply the acoustic stimuli to the intended left/right ear and to select the suitable electrode set that picks up the stimulated response to be recorded. The audio amplifier module has been designed and implemented to be able to generate audible sine wave at frequencies $500 \mathrm{~Hz}, 1 \mathrm{kHz}, 2 \mathrm{kHz}, 4 \mathrm{kHz}$, and $8 \mathrm{kHz}$. At each frequency the audible intensity of $5 \mathrm{~dB}$ SPL increment step ranges between $55 \mathrm{~dB}$ SPL to $110 \mathrm{~dB}$ SPL. They are the required frequency and intensity values to conduct hearing tests. The laboratory experiment has been conducted in a sound shielded chamber by using the standard TDH-39P headset that is clinically used to conduct hearing test. A Sound level meter (SLM) provided with selective filter is used to measure the audible output of the headset. The chamber noise had been measured before calibration conduction was done. It has value in the range 45 and $47 \mathrm{~dB}$ SPL (sound pressure level). The SLM was fixed $2 \mathrm{~cm}$ away in front of the active headset piece. The signal is software generated and applied through the NI-Daq board to the left/right Audio amplifier to be calibrated as shown in figure 3. The results of laboratory calibration for both audio amplifiers are listed in the table 1 . The $50 \mathrm{~dB}$ SPL is the minimum intensity that could be produced at all required frequencies except at $4000 \mathrm{~Hz}$ where the minimum intensity is 55dB SPL. $110 \mathrm{~dB}$ SPL is the highest intensity can be produced at frequencies 500 and $8000 \mathrm{~Hz}$ while $115 \mathrm{~dB}$ SPL is at $1000 \mathrm{~Hz}$ and $120 \mathrm{~dB}$ SPL at 2000 and $4000 \mathrm{~Hz}$.

The analog process module has been tested in three steps; the first has been dedicated to verify the filter's circuit functionality. It is Butterworth filter that passes all frequencies in the range $200 \mathrm{~Hz}$ and $3000 \mathrm{~Hz}$ and attenuates, drastically, the other components (especially the $50 \mathrm{~Hz}$ and its first and second harmonies). It is designed to have flat gain over its pass-band. The laboratory experiment resulted in the filtration response shown in figure 4. 


\begin{tabular}{|c|c|c|c|c|c|}
\hline Frequency & $\begin{array}{c}500 \\
\mathrm{~Hz}\end{array}$ & $\begin{array}{c}1000 \\
\mathrm{~Hz}\end{array}$ & $\begin{array}{c}2000 \\
\mathrm{~Hz}\end{array}$ & $\begin{array}{c}4000 \\
\mathrm{~Hz}\end{array}$ & $\begin{array}{c}8000 \\
\mathrm{~Hz}\end{array}$ \\
\hline $45 \mathrm{~dB}$ SPL & $\mathbf{X}$ & $\mathbf{X}$ & $\mathbf{X}$ & $\mathbf{X}$ & $\mathbf{X}$ \\
\hline $50 \mathrm{~dB}$ SPL & $\sqrt{ }$ & $\sqrt{ }$ & $\sqrt{ }$ & $\mathbf{X}$ & $\sqrt{ }$ \\
\hline $55 \mathrm{~dB}$ SPL & $\sqrt{ }$ & $\sqrt{ }$ & $\sqrt{ }$ & $\sqrt{ }$ & $\sqrt{ }$ \\
\hline $60 \mathrm{~dB}$ SPL & $\sqrt{ }$ & $\sqrt{ }$ & $\sqrt{ }$ & $\sqrt{ }$ & $\sqrt{ }$ \\
\hline $65 \mathrm{~dB}$ SPL & $\sqrt{ }$ & $\sqrt{ }$ & $\sqrt{ }$ & $\sqrt{ }$ & $\sqrt{ }$ \\
\hline $70 \mathrm{~dB}$ SPL & $\sqrt{ }$ & $\sqrt{ }$ & $\sqrt{ }$ & $\sqrt{ }$ & $\sqrt{ }$ \\
\hline $75 \mathrm{~dB}$ SPL & $\sqrt{ }$ & $\sqrt{ }$ & $\sqrt{ }$ & $\sqrt{ }$ & $\sqrt{ }$ \\
\hline $80 \mathrm{~dB}$ SPL & $\sqrt{ }$ & $\sqrt{ }$ & $\sqrt{ }$ & $\sqrt{ }$ & $\sqrt{ }$ \\
\hline $85 \mathrm{~dB}$ SPL & $\sqrt{ }$ & $\sqrt{ }$ & $\sqrt{ }$ & $\sqrt{ }$ & $\sqrt{ }$ \\
\hline $90 \mathrm{~dB}$ SPL & $\sqrt{ }$ & $\sqrt{ }$ & $\sqrt{ }$ & $\sqrt{ }$ & $\sqrt{ }$ \\
\hline $95 \mathrm{~dB}$ SPL & $\sqrt{ }$ & $\sqrt{ }$ & $\sqrt{ }$ & $\sqrt{ }$ & $\sqrt{ }$ \\
\hline $100 \mathrm{~dB}$ SPL & $\sqrt{ }$ & $\sqrt{ }$ & $\sqrt{ }$ & $\sqrt{ }$ & $\sqrt{ }$ \\
\hline $105 \mathrm{~dB}$ SPL & $\sqrt{ }$ & $\sqrt{ }$ & $\sqrt{ }$ & $\sqrt{ }$ & $\sqrt{ }$ \\
\hline $110 \mathrm{~dB}$ SPL & $\sqrt{ }$ & $\sqrt{ }$ & $\sqrt{ }$ & $\sqrt{ }$ & $\sqrt{ }$ \\
\hline $115 \mathrm{~dB}$ SPL & $\mathbf{X}$ & $\sqrt{ }$ & $\sqrt{ }$ & $\sqrt{ }$ & $\mathbf{X}$ \\
\hline $120 \mathrm{~dB}$ SPL & $\mathbf{X}$ & $\mathbf{X}$ & $\sqrt{ }$ & $\sqrt{ }$ & $\mathbf{X}$ \\
\hline
\end{tabular}

Table 1 Intensity calibration result for both left and right audio amplifiers. The $\sqrt{ }$ sign means the intensity has been produced while $\mathrm{X}$ sign means the intensity cannot be reached.

The second step has been conducted, on one side, to verify the Analog Process module ability to amplify signals of order of microvolts by using digital oscilloscope. On the other side it is to ascertain, experimentally, the whole system ability to function as audiometer before starting clinical experiments. To do it the laboratory test arrangement shown in figure 5 has been conducted. The measured output pure sine wave (on digital oscilloscope) of the function generator is minimum $200 \mathrm{mVp}$-p. So the signal has to be attenuated by $100 \mathrm{~dB}$ to be of $2 \mu \mathrm{Vp}$-p (to be of same amplitude order as ABR). The experiments have been conducted in unshielded laboratory environment.

For pure sine wave of $400 \mathrm{mVp}-\mathrm{p}$ and $2 \mathrm{kHz}$ is applied at the voltage divider input (upper signal on figure 6); the module output (Analog Process module) on oscilloscope (lower signal on figure 6) revealed around $200 \mathrm{mVp}-\mathrm{p}$ sine wave of $2 \mathrm{kHz}$ combined with its harmonics and modulated with low frequency signal of around $250 \mathrm{~Hz}$ as shown in figure 6 . The experiment has been conducted in unshielded laboratory environment. Figure (5) shows the input and output of applying a $2000 \mathrm{~Hz}$ signal of amplitude $400 \mathrm{mVp}$-p at the circuit input. 


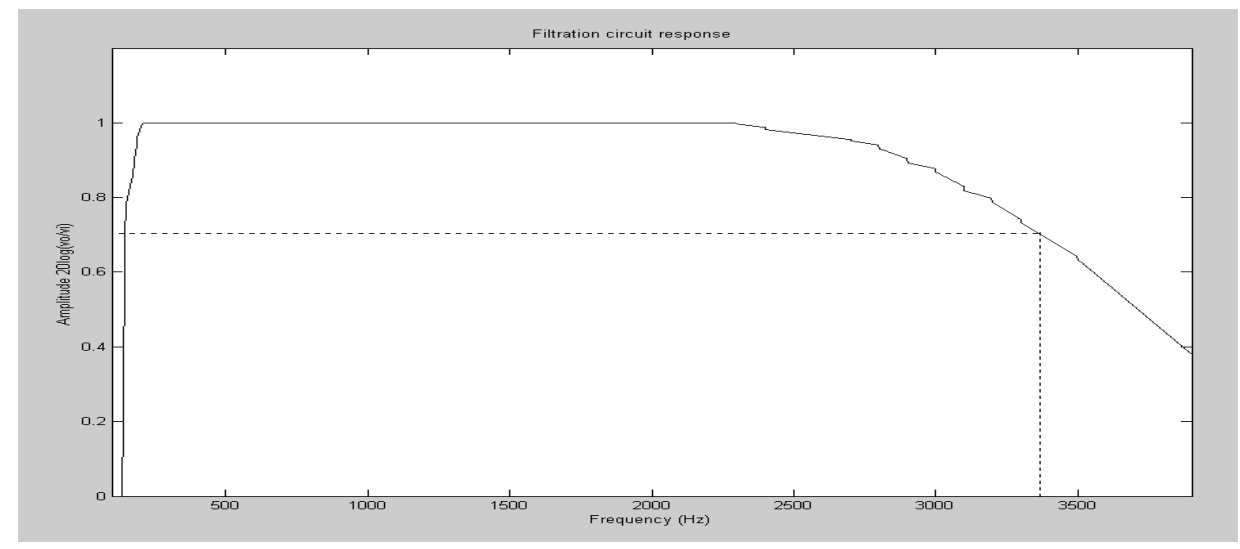

Figure 4 The measured response of filtration circuits. Horizontal axis is the frequency in $\mathrm{Hz}$ and vertical is the filter's gain in $\mathrm{dB}$.

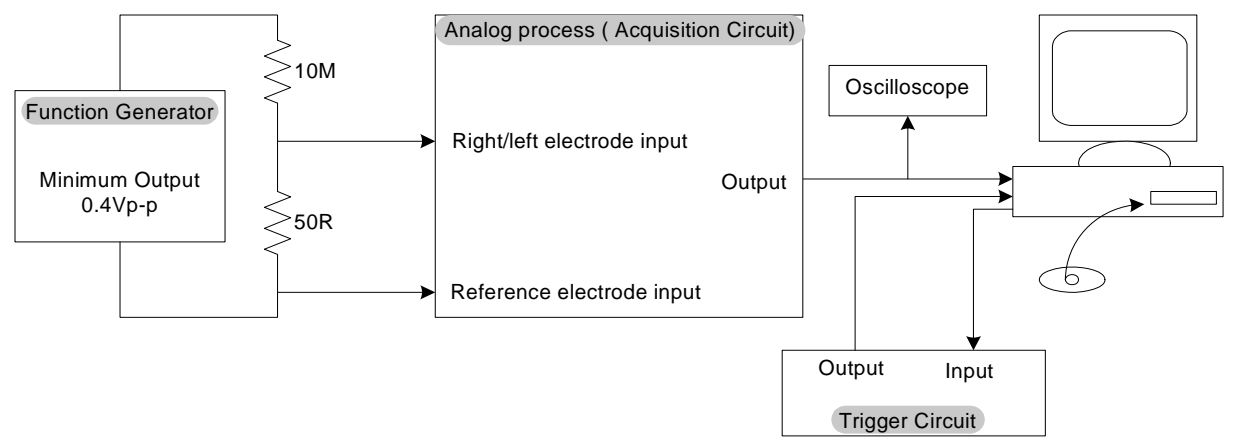

Figure 5 Block diagram of the laboratory test arrangement. The PCI NI-DAQ is not presented separately because it is slotted into the PC.

The module output is then connected to the PCI data acquisition board, and then the recorded output has been presented on the computer screen in both time and frequency domains. For both time and frequency domains, solid plot shown in figure 7 represents the recorded signal before any digital process. The frequency domain shows clearly the effects of low frequency components especially 200, 300 and $400 \mathrm{~Hz}$. They are the $50 \mathrm{~Hz}$ harmonics that are not attenuated analogically because they fall in our intended ABR frequency range. To extract the $2 \mathrm{kHz}$ signal, recorded signal has been digitally filtered and has been presented on the same plot as in figure 7 . To point out the $2 \mathrm{kHz}$ signal, a band pass digital filter of $1.9 \mathrm{kHz}$ and $2.2 \mathrm{kHz}$ cutoff frequencies has been used. Filtered signal is presented as dot-plot in the time domain and its line-base is shifted up by +4 for comparison and clarity purpose while, in frequency domain, it is shifted down and is presented as solid circle-marked plot. Both domains reveal that the Analog Process module is able to amplify its input 
signal by $10^{6}$ and also shows the low frequency noise effect and how the digital filtration enhances the intended signal.

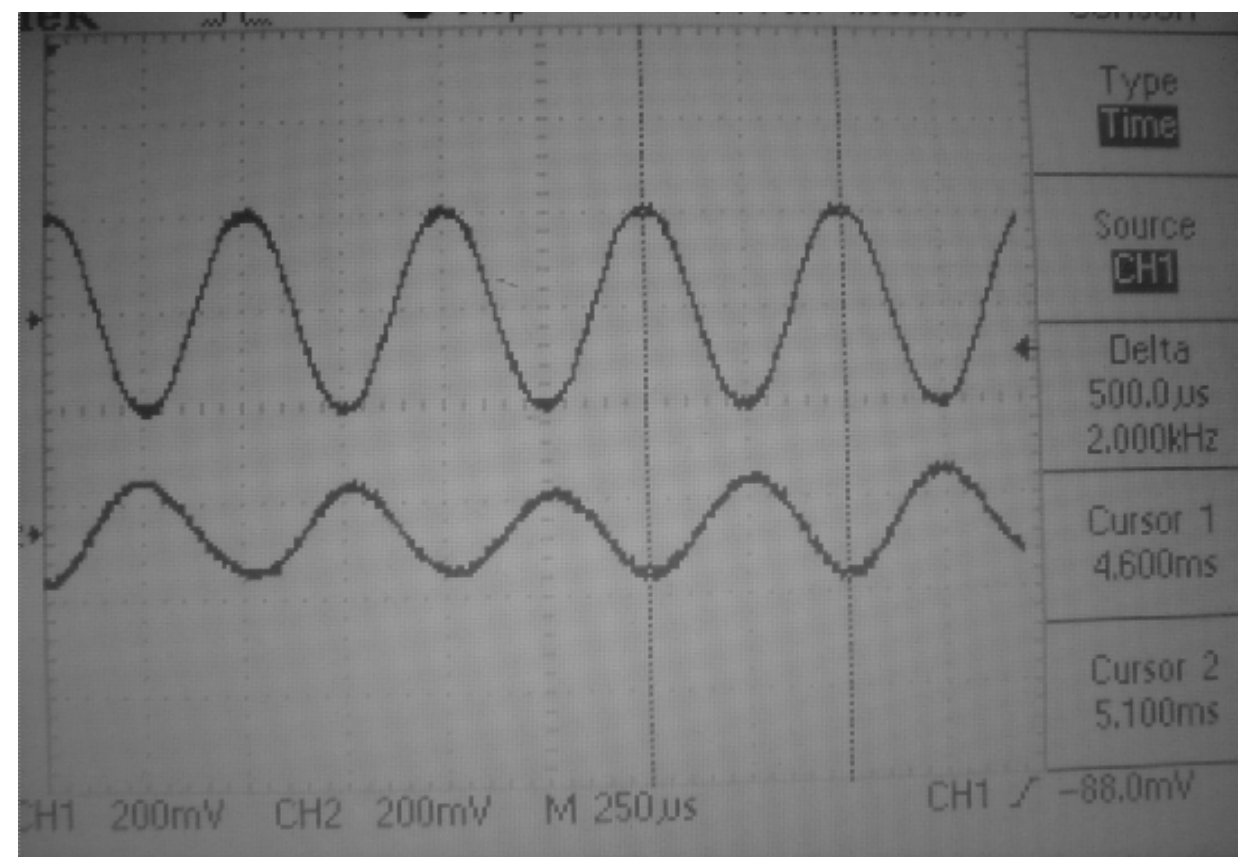

Figure 6 The test circuit input and the Analog Process module output on digital oscilloscope screen. The upper signal is measured at the voltage divider input that has output $10^{-6}$ times of its input. The lower signal is the Analog Process module output (200mV/Div). The same time axis (horizontal) is for both signals

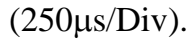

On the other side, a special trigger circuit has been designed and constructed to simulate ABR stimulation, recording, and to verify the signal extraction by using averaging method. The circuit, simply, generates a digital pulse that will trigger the acquisition board to start the data acquisition. Whenever a trigger takes place starting data acquisition, the same signal will be at the Analog Process module input and hence at the data acquisition analog input (figure 5). The software-controlled data acquisition card generates a signal to drive trigger circuit. The trigger circuit output-pulse is designed to force the same $2 \mathrm{kHz}$ signal to appear as a function generator output whenever a trigger takes place. In another word the software-generated signal that will control the trigger circuit is similar to the acoustic stimulus that will be generated and delivered to the hearing system. And the signal at the Analog Process module inputs (voltage divider output) is similar to the invoked ABR of a tested hearing system. Figure 8 shows the result of averaging 100 generated signals. The signal 
is a $400 \mathrm{mVp}-\mathrm{p}$ and $2 \mathrm{kHz}$ (measured on a digital oscilloscope) at the voltage divider input, and will be around $2 \mu \mathrm{Vp}$-p (calculated value) at the voltage divider output. The Analog Process module has been set to amplify its input signals by $25 * 10^{+3}$ before they are delivered to the analog input of the Data Acquisition board. The comparison of single picked up signal (figure 8) and the 100 averaged similar (to the single one) signals, clearly, shows that averaging enhanced the signal to noise ratio.

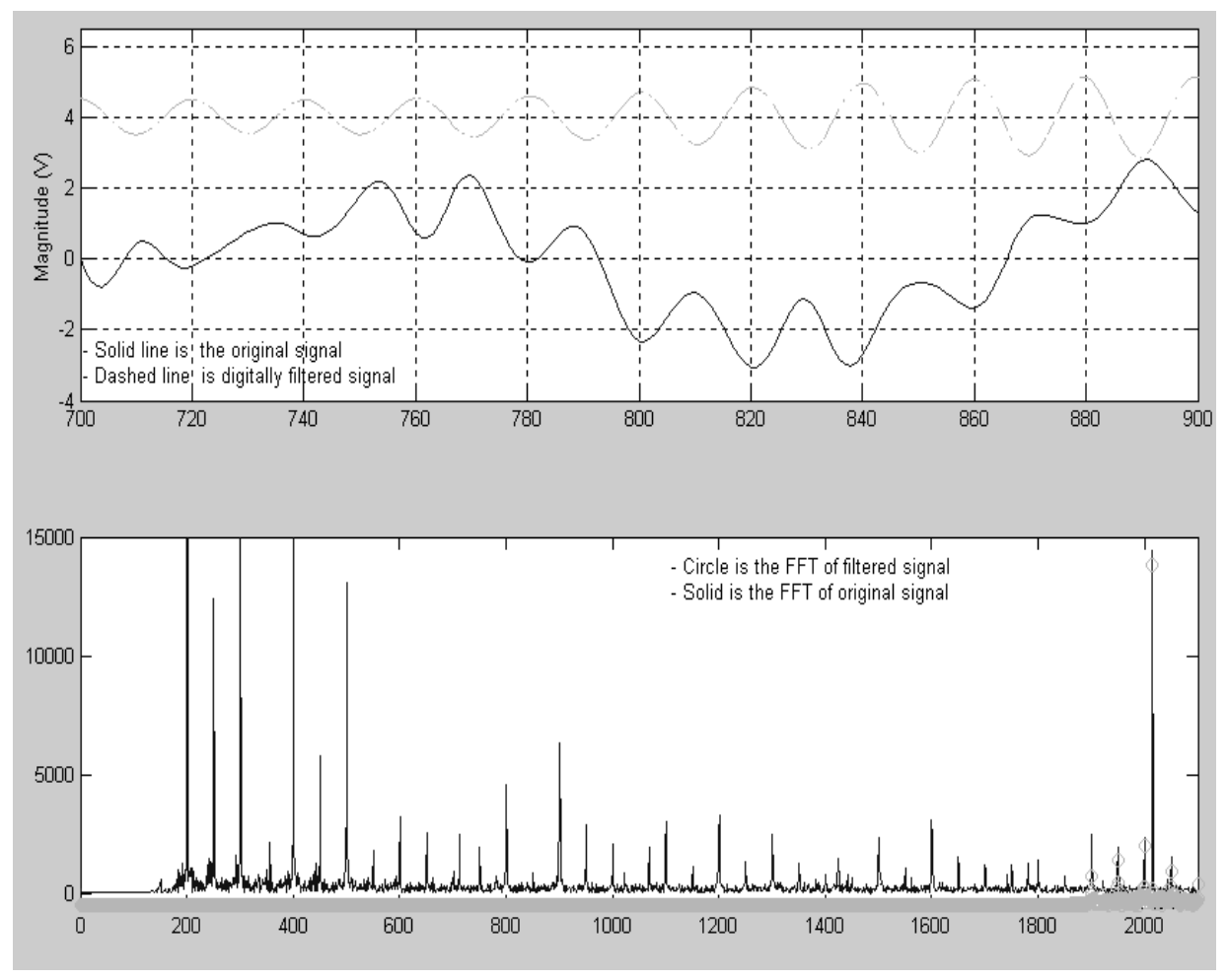

Figure 7 The recorded signal for $400 \mathrm{mVp}-\mathrm{p}$ and $2 \mathrm{kHz}$ sine wave input signal. Upper plots represent time domain signals where the vertical axis is in volts, while the horizontal axis is the number of samples. Time between successive samples is $25 \mu$ s (sampling frequency is $40 \mathrm{kHz}$ ). The lower plots represent the same signals in frequency domain where the horizontal axis is the frequency in $\mathrm{Hz}$ and the other is the amplitude (normalizing the values by 40000 gives the amplitude in volt). 


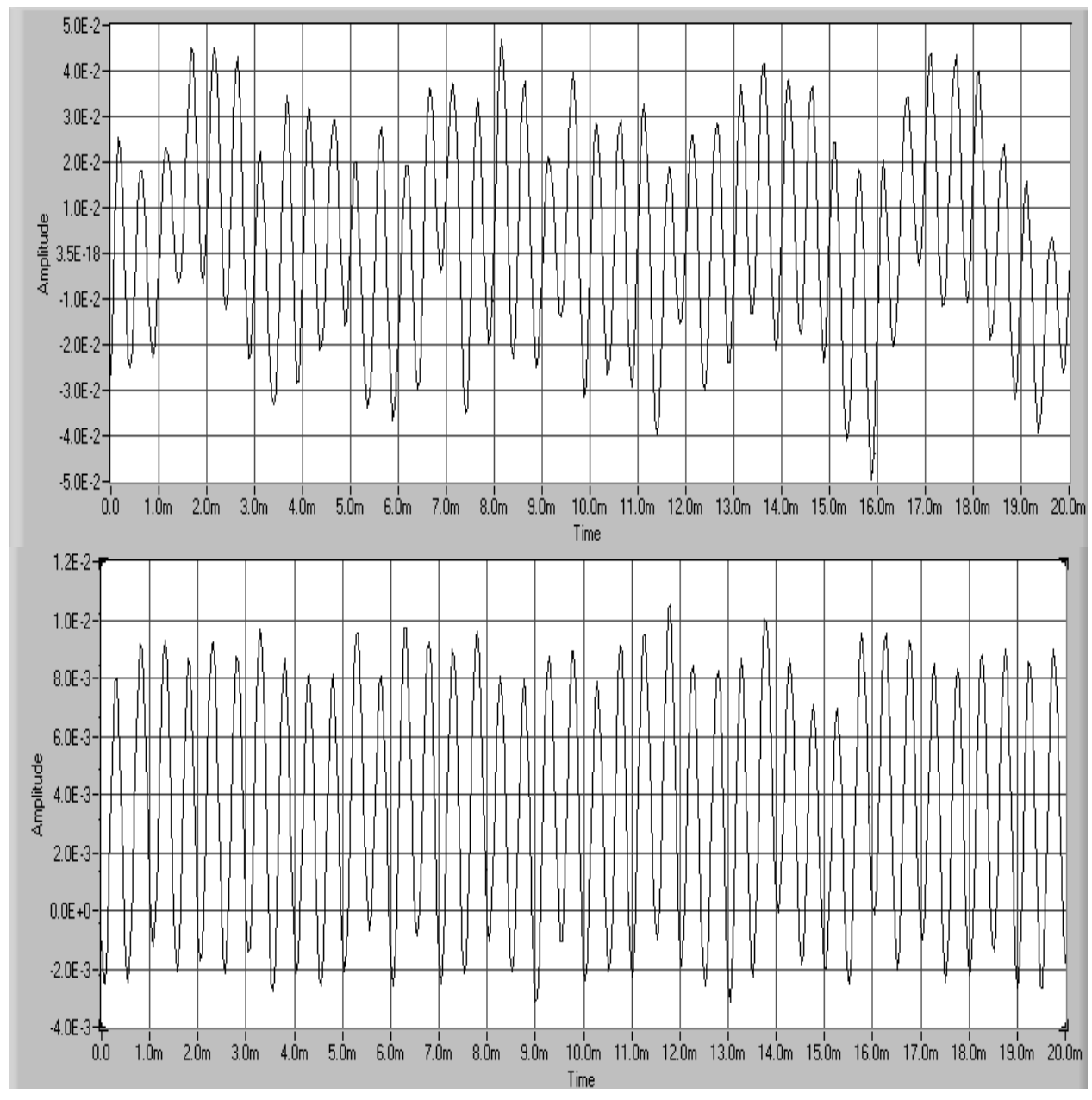

Figure 8 The attenuation effect of averaging method. The signal is acquired for $20 \mathrm{~ms}$ at sampling rate $25 \mathrm{kHz}$. The upper plot represents single generated and recorded signal. The lower signal represents the result of averaging 100 identical generated signals. The amplitude is measured in volts while the horizontal axis is the time in ms.

The research efforts have produced a developed Matlab program to generate falling chirp stimuli. The stimulus has been designed to synchronize the movements of all hearing cells that are sensitive to the frequencies the chirp stimuli include. Figure 9 represents two types of generated chirp stimuli that are fixed amplitude and falling chirp stimuli. Both stimuli are generated at sampling rate of $40 \mathrm{kHz}$ and they have the same frequency components that are $250 \mathrm{~Hz}$ through $4000 \mathrm{~Hz}$. The resulted chirp stimulus will be used to invoke the ABR biopotential during next experimental setup and clinical research. 


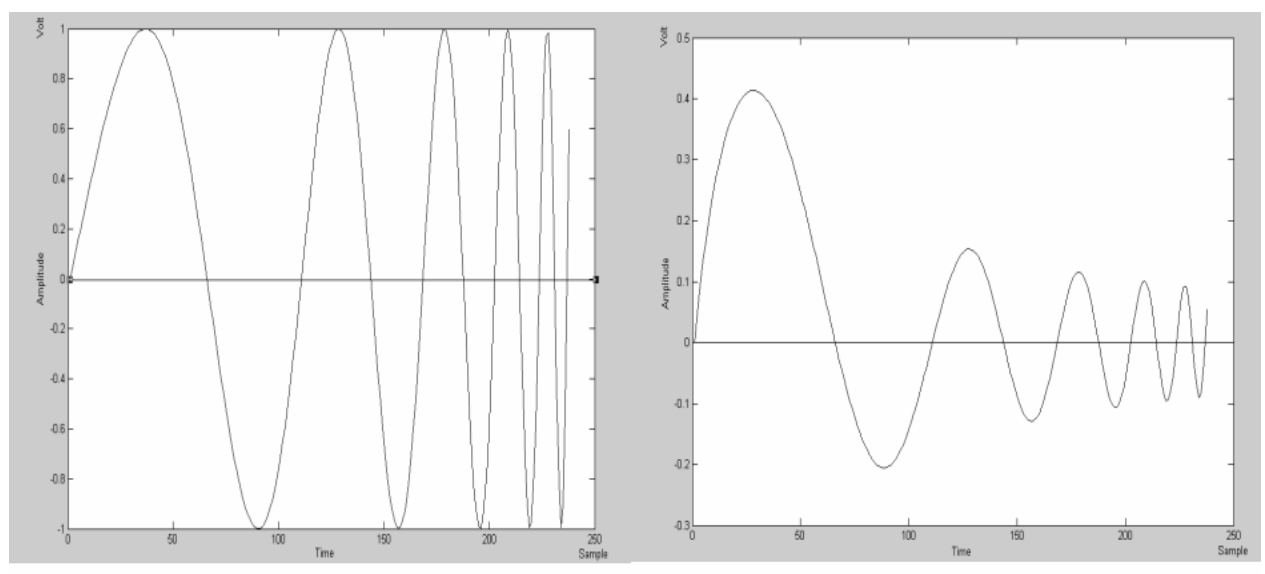

Figure 9 Chirp Stimulus. It contains frequencies of $250 \mathrm{~Hz}$ up to $4000 \mathrm{~Hz}$. Stimulus is generated at sampling rate of $40 \mathrm{kHz}$. The left plot represents fixed amplitude chirp signal and the right plot represents falling chirp stimulus of the same frequency contents. The vertical Axis is the amplitude in volt and the other is the time in the number of samples.

\section{$7 \quad$ Final Remarks}

1. The laboratory tests of Analog Process Module (figures 6,7) clearly reveal that any signal exists at the module input of $2 \mu \mathrm{V}$ amplitude will be of $2 \mathrm{~V}$ at the module output $\left(10^{+6}\right.$ time amplification). So our ABR biopotential that has amplitude of 0.1 to $10 \mu \mathrm{V}$ will be recordable and of 0.1 to $10 \mathrm{~V}$ amplitude at the module output.

2. Simulation of ABR invocation and extraction by using the averaging method revealed signal to noise enhancement as shown in figure 8 . The noise is attenuated by around $20 \mathrm{~dB}$ so there still exist $50 \mathrm{~Hz}$ harmonics effects.

3. The $50 \mathrm{~Hz}$ harmonics, especially the forth and fifth harmonics, are still have high effects in spite of good signal to noise ratio at the circuit input. This ratio is expected to be worse during clinical hearing test so there will be high necessity for advanced step of digital filtration.

4. The designed chirp stimulus that will be used to invoke the ABR bipotential is successfully generated.

5. Despite of communication flexibility the NI-DAQ board provides, there is still a necessity for additional circuit to improve the system functionality before conducting clinical experiments.

By overcoming various unexpected problems and system limitation problems we hope to achieve our target. With a little of hardworking we expect to be able 
to record and extract $\mathrm{ABR}$ at low frequencies. ABR is expected to consist of important information related to the whole cochlear sensorineural elements.

\section{$8 \quad$ References}

1. NIH Consens Statement Online, Early Identification of Hearing Impairment in Infants and Young Children (1993 Mar 1-3).

2. Michael E. Glassock III, M. D., F. A. C. S. \& C. Gary Jackson, M. D., F.A.C.S. \& Anne Forrest Josey, M. S., C. C. C. A., The ABR Handbook: Auditory Brainstem Response; second edition (1987).

3. ANSI working group S3: 76 (published May 1.st 1998): Performance Measures of Micro-processor or Computer Controlled Pure-Tone Audiometers, www.dhsc.com/ WG76.HTM.

4. $\quad$ Oliver Wegner, Torsten Dau \& Birger Kollmeier, On The Relationship Between Auditory Evoked Potentials And Psychophysical Loudness, Arbeitsgruppe Medizinische Physik, Carl von Ossietzky Universitat Oldenburg, Germany. Wegner.doc submitted to World Scientific 21.02.99: 15:59 2/5.

5. Author: Department of Anatomy. Tutorial, Auditory Pathway: Copyright (c) $2000 \quad$ University of $\quad$ Bristol http://137.222.110.150/calnet/Aud/page2.htm.

6. Webster \& John G. Medical, Instrumentation Application and Design, John willy \& Sons-USA (1998).

7. Aston \& Richard, Principles of Biomedical Instrumentation and Measurement, Merrill-USA (1990).

8. Ali AL-Afsaa \& Soegijardjo Soegijoko, Design and Implementation of PC-Based Audiometer Based on Brainstem Auditory Evoked Response, Jurnal Electronika dan Telecomunikasi, Volume III, Nomor3. 